\title{
FDG-Positron Emission Tomography/Computed Tomography from the Skull Base to Mid-thigh
}

National Cancer Institute

\section{Source}

National Cancer Institute. FDG-Positron Emission Tomography/Computed Tomography from the Skull Base to Mid-thigh. NCI Thesaurus. Code C137869.

Positron emission tomography combined with computed tomography of the region encompassing the skull base to the mid-thigh with the use of the contrast agent fludeoxyglucose F 18 (18F-FDG) to enhance the image. 